\title{
PERIODIC ANALYSIS OF SOLAR ACTIVITY AND ITS LINK WITH THE ARCTIC OSCILLATION PHENOMENON
}

 \\ ${ }^{1}$ Ocean University of China, 14-1'-601, 2117 Jinshui Road, Qingdao 266100, China; quweizhe@ ouc.edu.cn \\ ${ }^{2}$ Yantai Institute of Coastal Zone Research Chinese Academy of Sciences, China \\ Received 2013 August 1; accepted 2014 July 8; published 2014 November 13
}

\begin{abstract}
Based on spectrum analysis, we provide the arithmetic expressions of the quasi $11 \mathrm{yr}$ cycle, $110 \mathrm{yr}$ century cycle of relative sunspot numbers, and quasi $22 \mathrm{yr}$ cycle of solar magnetic field polarity. Based on a comparative analysis of the monthly average geopotential height, geopotential height anomaly, and temperature anomaly of the northern hemisphere at locations with an air pressure of $500 \mathrm{hPa}$ during the positive and negative phases of AO (Arctic Oscillation), one can see that the abnormal warming period in the Arctic region corresponds to the negative phase of $\mathrm{AO}$, while the anomalous cold period corresponds to its positive phase. This shows that the abnormal change in the Arctic region is an important factor in determining the anomalies of AO. In accordance with the analysis performed using the successive filtering method, one can see that the AO phenomenon occurring in January shows a clear quasi $88 \mathrm{yr}$ century cycle and quasi $22 \mathrm{yr}$ decadal cycle, which are closely related to solar activities. The results of our comparative analysis show that there is a close inverse relationship between the solar activities (especially the solar magnetic field index changes) and the changes in the $22 \mathrm{yr}$ cycle of the AO occurring in January, and that the two trends are basically opposite of each other. That is to say, in most cases after the solar magnetic index MI rises from the lowest value, the solar magnetic field turns from north to south, and the high-energy particle flow entering the Earth's magnetosphere increases to heat the polar atmosphere, thus causing the AO to drop from the highest value; after the solar magnetic index MI drops from the highest value, the solar magnetic field turns from south to north, and the solar high-energy particle flow passes through the top of the Earth's magnetosphere rather than entering it to heat the polar atmosphere. Thus the polar temperature drops, causing the AO to rise from the lowest value. In summary, the variance contribution rate of the changes in the quasi 110 yr century cycle and quasi $22 \mathrm{yr}$ decadal cycle for the $\mathrm{AO}$ reaches $62.9 \%$, indicating that solar activity is an important driving factor of the AO.
\end{abstract}

Key words: solar-terrestrial relations - Sun: activity

\section{INTRODUCTION}

The well-known quasi $11 \mathrm{yr}$ cycle, an approximately $110 \mathrm{yr}$ century cycle of solar activities, and quasi $22 \mathrm{yr}$ cycle of solar magnetic field polarity, are mostly expressions of qualitative analysis. In the process of studying the relationship between geophysical phenomena and solar activities, it is generally expected that there is a relatively strict arithmetic expression concerning solar activity cycle used to compare the solar activity cycle with geophysical phenomena. Based on spectrum analysis, we provide the arithmetic expressions of the quasi $11 \mathrm{yr}$ cycle, $110 \mathrm{yr}$ century cycle of relative sunspot numbers, and quasi $22 \mathrm{yr}$ cycle of solar magnetic field polarity. Based on the cyclic equations of relative sunspot numbers and solar magnetic field, we calculate the solar activity cycle and the solar magnetic field polarity cycle, which coincide with the actual curves of solar activities over the past $300 \mathrm{yr}$. The results prove that these arithmetic expressions accurately describe the decadal and centennial cycle characteristics of solar activities.

The effects of solar activities on the Earth's climate and atmospheric circulation have been studied by many scholars (Lean et al. 1995; Christensen \& Lassen 1991; Christensen 1993; Qu et al. 2004, 2012). The effects not only depend on the amount of solar radiation but also have an influence on the intensity and shape of the Earth's magnetic field through drastic changes in the solar magnetic field. This alters the ability of the Earth's magnetic field to trap charged particles, in turn causing the charged particles trapped by the Earth's magnetic field to travel between the North and South Poles around the magnetic force line of the Earth's magnetic field under Lorentz force, thereby releasing energy and heating the atmosphere. Although the amount of radiation does not increase significantly during solar activity, the ability of the Earth's magnetic field to trap the solar radiation of all of the bands doubles in scale. Therefore, the increase in the Earth's temperature is much greater than the increase in the amount of solar radiation. This signifies that solar activity exerts effects on the climate and physical environment of the Earth through changes in both solar radiation and the magnetic field, the latter of which plays the greater role.

Establishing the time series for the magnetic index (MI) of the solar magnetic field provides a very reliable reference for specifically studying the effects exerted by the polarity cycle of sunspot and magnetic field intensity on geophysical events. It also aids us in studying and understanding solar activity and its methods of affecting the environment and physical events of the Earth from an angle of changes in the solar magnetic field. The current paper investigates the effects on Arctic Oscillation exerted by solar activity from the two aspects of changes in solar radiation and those in the solar magnetic field. Changes in the solar magnetic field and the effects of those changes on many geophysical events are becoming increasingly more evident but research delving deeper into their 
Table 1

Power Spectrum and Test Spectrum of the Relative Sunspot Number index from 1700 to 2010 (Reliability $\alpha=0.05$ )

\begin{tabular}{lccccccccc}
\hline \hline Wave Number & 0 & 1 & 2 & 3 & $\mathbf{4}$ & 5 & 6 & 7 & 8 \\
\hline Wavelength & $\infty$ & 44 & 22 & 14 & $\mathbf{1 1}$ & 8 & 7 & 6 & 5 \\
Spectral density & 0.254 & 0.084 & 0.032 & 0.006 & $\mathbf{0 . 5 4 9}$ & 0.138 & 0.003 & 0.015 & 0.015 \\
Test spectrum & 0.758 & 0.490 & 0.238 & 0.129 & $\mathbf{0 . 0 7 9}$ & 0.054 & 0.039 & 0.030 & 0.023 \\
Amplitude & 2.000 & 7.085 & 4.177 & 5.961 & $\mathbf{3 0 . 2 3}$ & 4.323 & 1.766 & 1.964 & 2.412 \\
Initial phase angle & 0.000 & 0.110 & 0.667 & -1.27 & $\mathbf{- 2 . 1 9}$ & -0.27 & 0.738 & 0.951 & 1.510 \\
\hline
\end{tabular}

Table 2

Power Spectrum and Test Spectrum of the Relative Sunspot Number Index from 1700 to 2010 (Reliability $\alpha=0.05$ ) Without Short-Wave Impact

\begin{tabular}{lccccccccc}
\hline \hline Wave Number & 0 & 1 & $\mathbf{2}$ & 3 & 4 & 5 & 6 & 7 & 8 \\
\hline Wavelength & $\infty$ & 220 & $\mathbf{1 1 0}$ & 73 & 55 & 44 & 36 & 31 & 22 \\
Spectral density & 0.114 & 0.101 & $\mathbf{0 . 3 7 5}$ & 0.064 & 0.106 & 0.075 & 0.029 & 0.031 & 0.002 \\
Test spectrum & 1.172 & 0.504 & $\mathbf{0 . 1 8 6}$ & 0.091 & 0.053 & 0.034 & 0.024 & 0.018 & 0.009 \\
Amplitude & 2.000 & 8.632 & $\mathbf{1 5 . 1 7}$ & 3.058 & 6.838 & 5.195 & 4.832 & 0.964 & 0.947 \\
Initial phase angle & 0.000 & 0.710 & $\mathbf{- 1 . 3 9}$ & -0.67 & -2.01 & -1.87 & 1.338 & 0.151 & 1.910
\end{tabular}

methods of contact began decades ago (Qu et al. 2003, 2005; Zhang et al. 1998; Zhang \& Xiao 2000; Shi \& Wang 1994; Dong 1997; Xia \& Jin 2001).

Arctic Oscillation (AO) is an anti-phase oscillation phenomenon related to pressure in the areas near the North Pole and mid-latitude pressure field around the pole, and it is the main type of atmospheric motion. In addition, the main variables accounting for atmospheric motion in the northern hemisphere all possess the $\mathrm{AO}$ oscillation mode. Atmospheric climate anomalies related to abnormal changes in the AO have moved far beyond the polar regions, ranging over the entire hemisphere. AO shows an equivalent barotropic characteristic from low to high altitudes; moreover, with an increase in height, the north-south oscillation form becomes clearer and the variance contribution rate becomes much greater; thus the oscillation becomes the dominant form of atmospheric motion. $\mathrm{AO}$ has a very wide frequency domain, as both the interannual and decadal scales are very evident. Therefore, it is of great significance to study the regularities of the changes in the AO, especially the decadal variation characteristics, as they have become an important component of studies regarding unusual climate change.

What is the forced source driving the AO phenomenon? Some studies have noted effects of many factors such as the fluctuation of sea ice and the abnormality of the SST field. The authors of this study believe that these are directly related phenomena. The abnormalities of sea ice and sea surface temperature are the results of temperature anomalies; thus the abnormal changes in temperature, especially the changes in polar temperature, are a trigger of AO. The most important factor affecting the changes in polar temperature is solar activity; therefore we may link $\mathrm{AO}$, polar temperature, and solar activity together.

This paper uses the successive filtering method. Previous studies have shown that $\mathrm{AO}$ has a significant cycle consistent with stable solar activity, which furthers our understanding of the driving factors of the $\mathrm{AO}$ phenomenon. $\mathrm{AO}$ data have been obtained from http://www.atmos.colostate. edu/ao/Data/ao_index.html.

\section{PERIODIC FEATURES OF SOLAR ACTIVITY}

This paper uses relative sunspot numbers and a periodic discussion of solar activity based on the Sun's magnetic field magnetic index time series, and we discuss the periodic polarity cycle of the solar magnetic field.

\subsection{Decadal and Century Cycles of Solar Activities}

Table 1 shows the parameters of the spectrum analysis of the relative sunspot numbers. It can be seen that the $11 \mathrm{yr}$ cycle spectral value of 0.549 of the fourth wave is clearly higher than the spectral value of 0.079 shown in the test results, and the $11 \mathrm{yr}$ cycle spectral value is the spectral peak of all the subordinate frequency domains. Therefore, the quasi $11 \mathrm{yr}$ cycle is the most clearly identifiable cycle of solar activity.

In order to investigate the century cycle characteristics of solar activities, we conducted $17 \mathrm{yr}$ moving average processing over the relative sunspot number sequence so as to remove the decadal cycle and short-wave impact. The analysis results of the time sequence spectrum of the relative sunspot number without short-wave impact are shown in Table 2. It may be seen that the $110 \mathrm{yr}$ cycle spectral value of 0.375 in the second wave is clearly higher than the spectral value of 0.186 shown in the test results, and it is the spectral peak of all the frequency domains. Therefore, the quasi $110 \mathrm{yr}$ is the most clearly identifiable century cycle of solar activities.

Clearly, the $11 \mathrm{yr}$ cycle of solar activity overlies the $110 \mathrm{yr}$ cycle, and the two cycles can be written using a single equation $C_{\mathrm{R} 11011}(i)$ :

$$
\begin{aligned}
C_{R 11011}(i)= & \sum_{i=1}^{m} A_{l} \sin \left(\frac{2 \pi}{T_{l}} i+\varphi_{l}\right) \\
= & 15.17 \sin \left(\frac{2 \pi}{110} i-1.39\right) \\
& +30.23 \sin \left(\frac{2 \pi}{11} i-2.19\right)(i=1,2, \ldots \ldots 311)
\end{aligned}
$$




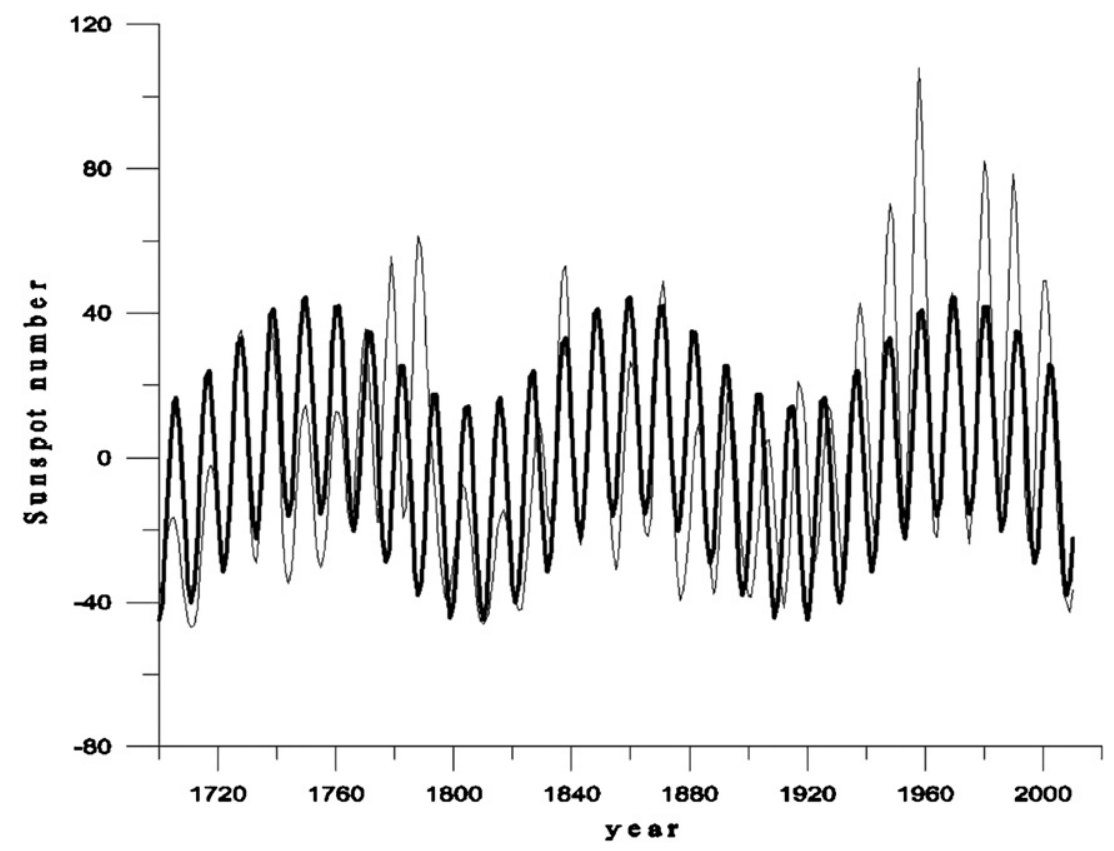

Figure 1. Five-year moving average curve of the relative sunspot number anomaly (thin line) and composite curve of the $110 \mathrm{yr}$ and $11 \mathrm{yr}$ cycles (black line).

Table 3

Power Spectrum and Test Spectrum of the Magnetic Index of the Sun from 1700 to 2010 (Reliability $\alpha=0.05$ )

\begin{tabular}{lccccccccc}
\hline \hline Wave Number & 0 & 1 & 2 & 3 & $\mathbf{4}$ & 5 & 6 & 7 & 8 \\
\hline Wavelength & $\infty$ & 88 & 44 & 29 & $\mathbf{2 2}$ & 17 & 14 & 12 & 11 \\
Spectral density & 0.072 & 0.009 & 0.028 & 0.015 & $\mathbf{0 . 8 2 9}$ & 0.118 & 0.019 & 0.031 & 0.009 \\
Test spectrum & 1.740 & 0.444 & 0.138 & 0.064 & $\mathbf{0 . 0 3 7}$ & 0.024 & 0.012 & 0.018 & 0.009 \\
Amplitude & 2.000 & 3.87 & 0.91 & 7.73 & $\mathbf{6 1 . 2 3}$ & 5.62 & 5.33 & 0.76 & 0.69 \\
Initial phase angle & 0.000 & \multirow{2}{*}{1.710} & -1.09 & -1.67 & $\mathbf{- 3 . 4 7}$ & -1.07 & 1.738 & 0.951 & 1.110 \\
\hline
\end{tabular}

In order to testify whether or not Formula (1) accurately reveals the periodical change of solar activity, in Figure 1. We draw the five-year moving average curve of the relative sunspot number anomaly from 1700 to 2010 (thin line). From the blue line in the figure, it may be seen that the $11 \mathrm{yr}$ cycle of solar activity is very clear, and that the amplitudes of the $11 \mathrm{yr}$ cycle are strong in some years and weak in others, as is revealed in the spectrum analysis. The $110 \mathrm{yr}$ century cycle is shown by the first item on the right-hand side of Formula (1). In Figure 1 we draw the composite cycle of the $110 \mathrm{yr}$ century cycle and 11 yr decadal cycle expressed in Formula (1) (bold line). Comparing the red and blue anomaly curves of the relative sunspot number, the theoretical curves of the quasi $11 \mathrm{yr}$ cycle deviate from the actual curves in only the third and fourth periods (from 1775 to 1790), and almost all of the theoretical cycles conform with the actual cycles, especially from the 1950s to the present, where the theoretical and actual phases conform well. We should note that the theoretical curves are calculated beginning from 1700 , i.e., after running for $300 \mathrm{yr}$, and the theory and actuality show no clear deviation. This proves that the solar activity cycle operates according to existing laws and that Formula (1) is correct.

Through analysis of variance, it can be seen that the rate of contribution of the $11 \mathrm{yr}$ cycle to the relative sunspot number anomaly from 1700 to 2010 is $55.3 \%$, and that the rate of contribution of the $110 \mathrm{yr}$ cycle is $7.3 \%$; together the two reach a rate of construction of $62.6 \%$. This shows that the periodicity of solar activity described in Formula (1) is the basic feature of the solar activity.

\subsection{Quasi 22 yr Cycle of Solar Magnetic Field Polarity}

The exponential time sequence of the solar magnetic field records the history of the sunspot magnetic cycle, and the spectrum analysis results are shown in Table 3. From the table, it may be seen that the $22 \mathrm{yr}$ cycle spectral value of 0.829 in the fourth wave is clearly higher than the spectral value of 0.037 shown in the test results, and that the $11 \mathrm{yr}$ cycle spectral value is the spectral peak of all of the subordinate frequency domains. Therefore, the quasi $22 \mathrm{yr}$ cycle is the most clearly identifiable cycle of solar magnetic polarity. Further study shows that no longer century cycles of exponential time sequence exist.

Based on the amplitude 61.23 and initial phase angle -3.47 of the quasi $22 \mathrm{yr}$ cycle provided in Table 3, the expression of the quasi 22 yr cycle $C_{\text {MI22 }}(i)$ of the solar magnetic field polarity exponential time sequence is as follows:

$C_{M I 22}(i)=61.23 \times \sin \left(\frac{2 \pi}{22} i-3.47\right)(i=1,2, \ldots \ldots 311)$.

In order to test whether or not Formula (2) accurately reveals the periodic changes of the solar magnetic field polarity cycle, in Figure 2 we draw the five-year moving average curve (thin line) of the solar magnetic polarity exponents from 1700 to 


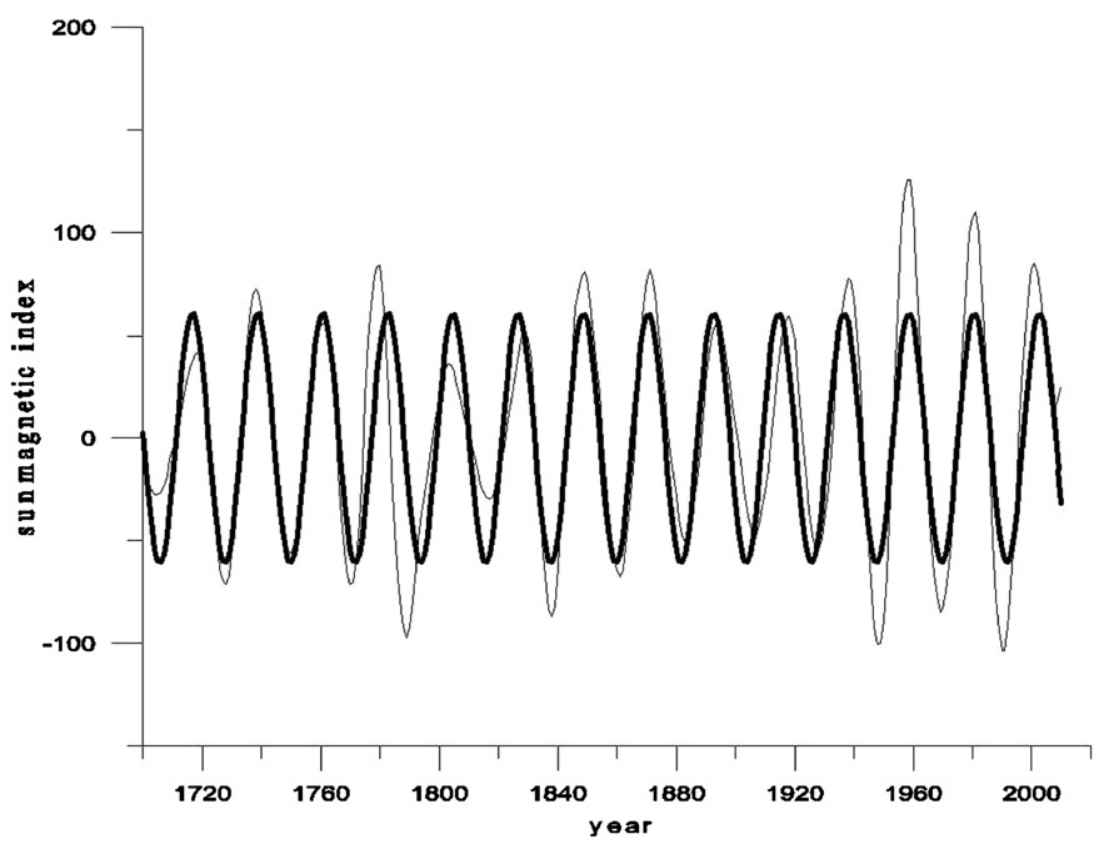

Figure 2. Five-year moving average of solar magnetic field polarity exponents (thin line) and $22 \mathrm{yr}$ cycle curve (bold lines).

2010. From the blue line in the figure, it may be seen that the quasi 22 yr cycle of the solar magnetic polarity exponent is very clear, and that the century cycle of the polarity exponential time sequence is not very clear. In the same figure, we also drew the 22 yr cycle expressed in Formula (2) (bold line); comparing the red cycle curve with the blue solar magnetic field exponent curve, the theoretical cycle curve is shown to basically conform with the actual curve. We should note that the theoretical cycle is calculated beginning from the year 1700, i.e., after running for $300 \mathrm{yr}$; the theory and the actuality show no clear deviation. This proves that the solar magnetic polarity cycle operates according to existing laws and that Formula (2) is correct.

Through analysis of variance, it may be seen that the rate of construction of the $22 \mathrm{yr}$ cycle to the solar magnetic polarity exponent from 1700 to 2010 is $70.9 \%$, i.e., the solar magnetic field polarity cycle described in Formula (2) is merely the basic feature of the solar magnetic field changes.

\section{ATMOSPHERIC CIRCULATION CHARACTERISTICS REFLECTED BY AO}

Arctic Oscillation (AO), also known as the northern hemisphere annular mode (NAM), represents the inverse oscillation characteristics reflected by related signals in the polar and central-latitude atmosphere regions of the northern hemisphere. However, there are no specific atmospheric circulation characteristics. In order to further understand the atmospheric circulation reflected by $\mathrm{AO}$ and explore its relevant factors, in this study, the atmospheric temperature-pressure field structural level is observed, which shows a clear physical meaning. In addition, the month chosen was that when the oscillation form was at its clearest and the NAM time factor was at its largest. At this time, the distribution characteristics of large-scale atmospheric circulation in the northern hemisphere portray the basic reflection of $\mathrm{AO}$ in the atmospheric circulation during the positive phase period of AO. This is also the entry point for us to study the formation mechanisms of AO in terms of atmospheric circulation.

\subsection{Differences of Atmospheric Circulation Characteristics at Positive and Negative Phase Periods of AO at Central and High Latitudes of the Northern Hemisphere $500 \mathrm{hPa}$}

Due to the strong barotropic structure of AO, the north-south oscillation form of $\mathrm{AO}$ is clear. Thus, for our analysis, we choose the $500 \mathrm{hPa}$ level, which represents the mid-troposphere to a high degree. This article uses the January AO to analyze the relation between $\mathrm{AO}$ and the solar activity for the following three reasons. (1) The AO phenomenon appears in January during the winter season in particular (Thompson \& Hurrell 2002). (2) There is no direct solar radiation in the North Pole area in January, so it is possible to reduce the disturbance to the motion of the atmosphere caused by direct solar radiation. The impact of the solar magnetic field polarity cycle on $\mathrm{AO}$ is exaggerated. (3) The article may be too long if the July analysis is added.

From the time series of AO in January from 1889 to 2013, we may determine that the maximum value of the AO positive phase occurs in January 1993 (monthly anomaly: 4.162). In other words, regardless of whether being compared with various historical periods of $\mathrm{AO}$ or other oscillation modes, the characteristics of the annular mode at this time are the clearest. Therefore, this is the best time to study the causes of $\mathrm{AO}$ in terms of atmospheric circulation by investigating the characteristics of atmospheric circulation at this time in the northern hemisphere.

First, the monthly average of $500 \mathrm{hPa}$ geopotential height in the northern hemisphere (north of $20^{\circ} \mathrm{N}$ ) in 1993 January is acquired, as shown in Figure 3(a). As we may see from Figure 3(a), near the Arctic pole there is a deeply developed polar vortex; the central latitudes $\left(20-50^{\circ} \mathrm{N}\right)$ surrounding the Arctic pole are smooth trough-ridge fluctuations. The three shallow high-pressure ridges lie, respectively, in the northern part of the Asian continent, the northwestern part of the North American continent, and on the Atlantic coast of Africa and Europe. When AO is in the positive phase, the barometric gradient which the central latitudes direct at the polar 

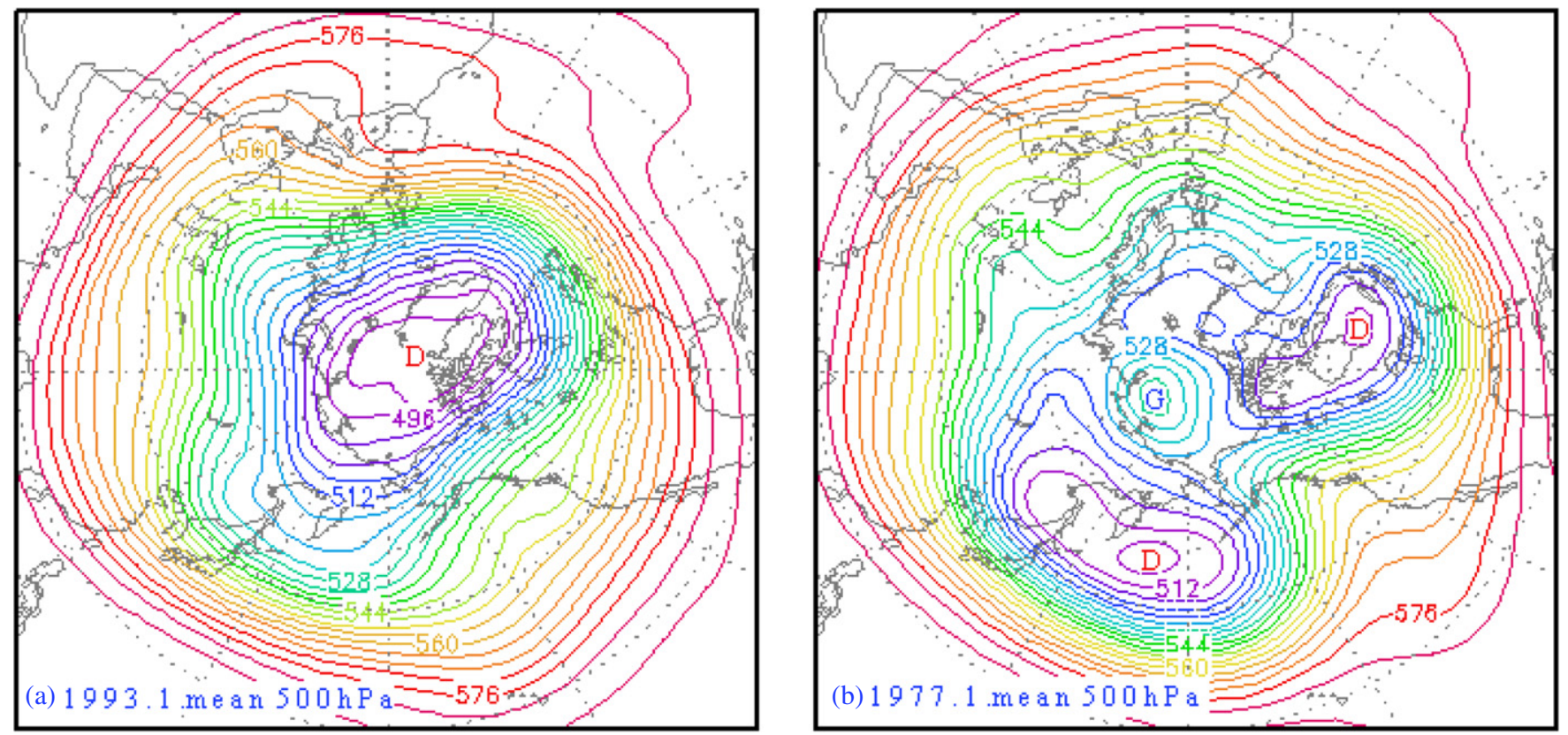

Figure 3. Geopotential height distribution diagram of the northern hemisphere $500 \mathrm{hPa}$ in January. (a) 1993 January and (b) 1997 January.

region is very large; zonal circulation prevails in the central and high latitudes, which restricts the cold air in the polar regions from expanding southward and the frequency of cold waves is lower, thus causing the northern hemisphere to have fewer frosts.

The maximum value of the $\mathrm{AO}$ negative phase during the period from 1889 to 2013 occurs in 1940 January, the monthly anomaly of which is -3.451 , and the second largest occurs in 1977 January, with a monthly anomaly of -3.279 . Considering the fact that the density representativeness of the meteorological data from the 1940s is inferior to that from the 1970s and that the maximum value of the AO negative phase is -3.279 in 1977 January, we selected 1977 January as the representative month for conducting our research.

Next we acquired the monthly average of the geopotential height in the northern hemisphere $500 \mathrm{hPa}$ in 1977 January, as shown in Figure 3(b). From the figure it may be seen that the polar vortex shifts out of the polar region after filling and splitting and what takes over is a polar closed high-pressure center. At the typical trough areas of the North American Great Lakes and East Asia, two deep, cold vortices are formed. Affected by this, the temperatures were abnormally low in the North American and Eurasian continents in 1977 January.

Only the Atlantic coast of Europe and the Pacific coast of North America were affected by the warm and wet airflow in front of the ridge, causing the climate there to be mild and rainy.

$\mathrm{AO}$ reveals the fluctuation law of pressure fields of polar regions and central latitude areas of the northern hemisphere. The following are the distribution characteristics of anomaly fields at the positive and negative phase periods of $\mathrm{AO}$ in the polar regions and central latitudes $500 \mathrm{hPa}$ of the northern hemisphere.

From Figure 4(a), it may be seen that the air pressure is abnormally low in the polar regions at the positive phase of AO in January 1993, at which time the negative anomaly falls below $-20 \mathrm{hPa}$; the area of $40^{\circ} \mathrm{N}$ surrounding the polar regions is the positive area and the pleions of the African and European continent exceed $+14 \mathrm{hPa}$. Therefore, the development level of
AO in January mainly depends on the polar vortex and highpressure ridge of the central latitudes; when the polar vortex and the high-pressure ridge of the central latitudes grow abnormally, the AO is strong; the air pressure of the polar vortex active area drops and that of the high-pressure area in the central latitudes rises. Therefore, the barometric gradient between the central and high latitude areas increases greatly, while the west wind enhances and zonal circulation prevails.

From Figure 4(b) it may be seen that the air pressure of the arctic region $500 \mathrm{hPa}$ is abnormally high during the negative phase of AO in 1977 January, at which time the positive anomaly exceeds $+40 \mathrm{hPa}$, the area of $40^{\circ} \mathrm{N}$ surrounding the polar regions is the negative area, and the pleions of the North Atlantic Ocean fall below $-20 \mathrm{hPa}$. Based on these observations, it may be seen that when the polar vortex and the high-pressure ridge of the central latitudes weakens abnormally in January, the AO also weakens. The air pressure of the polar vortex active area rises and that of the high-pressure area in the central latitudes drops; thus the barometric gradient between the central and high latitude areas decreases greatly, the west wind weakens, zonal circulation collapses, and meridional circulation prevails, and the cold and warm air exchange in the north and south is active.

\subsection{Difference of Abnormal Distribution Characteristics \\ During the Period of Positive and Negative Phases of AO in the Central and High Latitudes of the Northern Hemisphere $500 \mathrm{hPa}$}

Figure 5 shows the thermic anomaly distribution characteristics during the period of the positive and negative phases of $\mathrm{AO}$ in the central and high latitudes of the northern hemisphere $500 \mathrm{hPa}$. Figure 5(a) is the thermic anomaly distribution diagram during the positive phase of $\mathrm{AO}$ to the north of $20^{\circ} \mathrm{N}$ in the northern hemisphere $500 \mathrm{hPa}$ in 1993 January. Then the temperature of the polar regions is abnormally low, which leads to the decline of the isobaric surface and the abnormal deepening of the polar cyclone. The temperature of the central and high latitudes, besides those in the polar regions, shows 

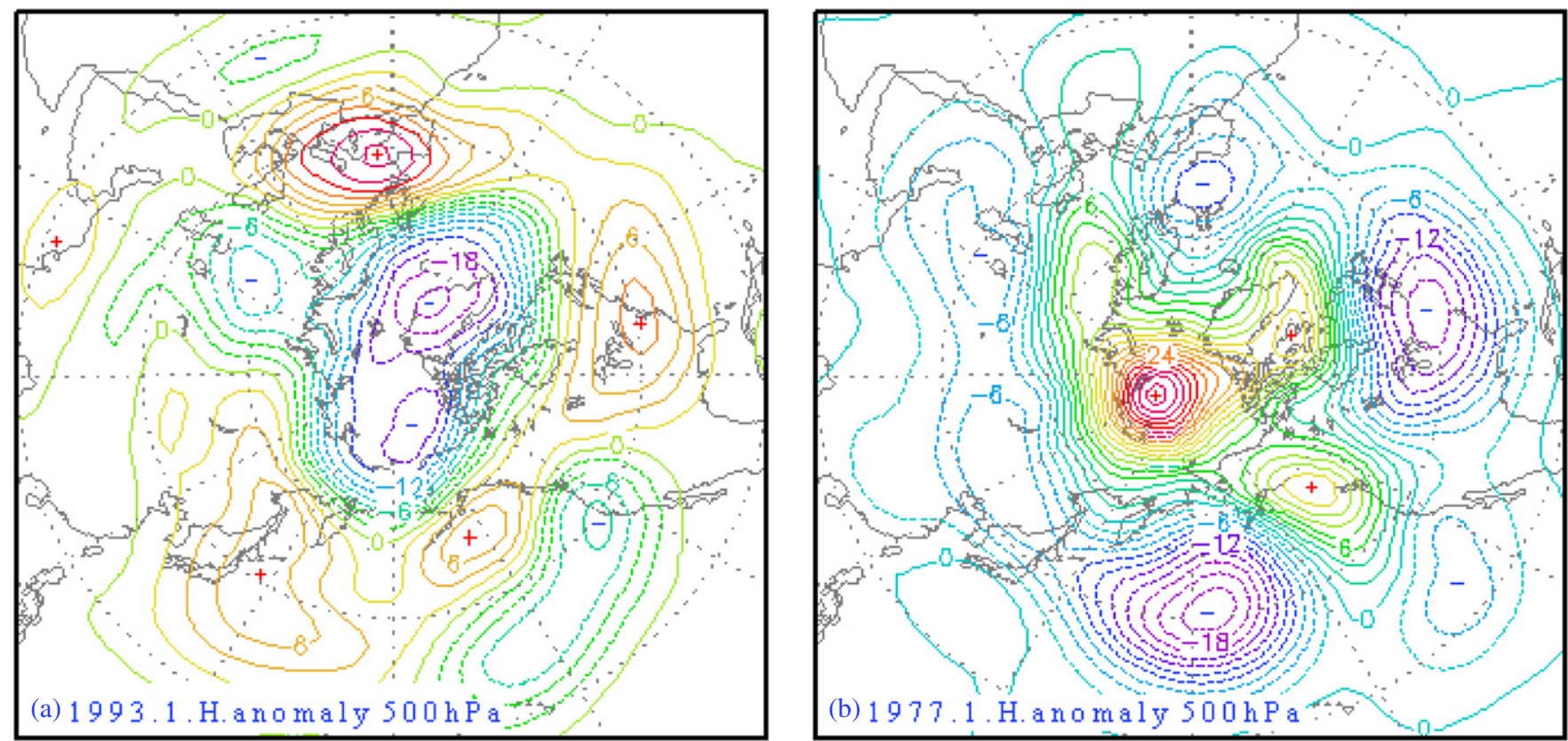

Figure 4. Anomaly distribution diagram of the northern hemisphere 500 hPa hgt. (a) 1993 January. (b) 1997 January.
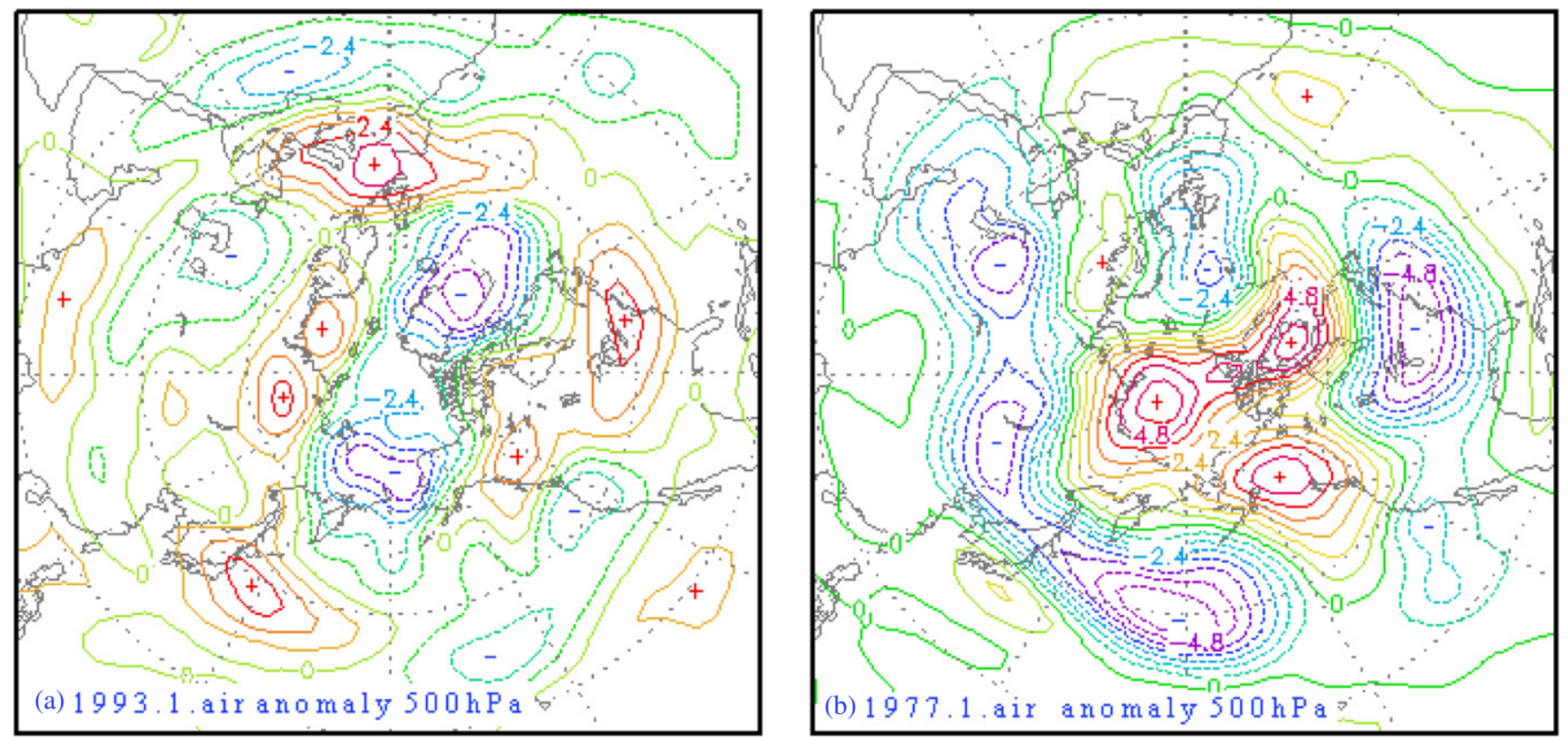

Figure 5. Distribution diagram of temperature departure in the northern hemisphere 500 hPa. (a) 1993 January. (b) 1977 January.

a predominantly positive anomaly in the geopotential height field; this positive anomaly roughly corresponds to the locations of three shallow high-pressure ridges in the northern Asian continent, northwestern North American continent, and the Atlantic coast of Africa and Europe (see Figure 3(a). After comparing with Figure 4(a), we may see that the symbol distribution of the temperature difference roughly shows a one-to-one correspondence with that of the geopotential height anomaly $500 \mathrm{hPa}$ in 1993 January, which signifies that the atmosphere is basically in a state of positive pressure.

Figure $5(\mathrm{~b})$ is the abnormal distribution diagram during the negative phase of $\mathrm{AO}$ to the north of $20^{\circ} \mathrm{N}$ in the northern hemisphere $500 \mathrm{hPa}$ layer in 1977 January. The temperature is abnormally high at the polar regions and abnormally low in the central latitudes. The abnormally high temperature in the polar regions causes the polar vortex to shift out of the polar regions after filling and splitting, and what takes over is a polar closed high-pressure center. The abnormally low temperature in the central latitudes causes the geopotential height to decline abnormally, which is the main cause of the formation of two deep, cold vortices in the trough area of the North American Great Lakes and the low-pressure area of the East Asia deep trough-Aleutian. After comparing this figure with Figure 4(b), it may can seen that the symbol distribution of the temperature difference is roughly in one-to-one correspondence with that of the geopotential height anomaly in 1977 January $500 \mathrm{hPa}$. 
This shows the rapid adaptation of geopotential height caused by the change in the atmosphere temperature field.

After comparing the positive and negative phases of $\mathrm{AO}$, it may be observed that there are three major differences in the temperature field.

1. The most significant difference during the periods of the positive and negative phases of $\mathrm{AO}$ is that the temperature changes from meions to pleions. The polar vortex is a deep, cold low-pressure circulation system, and the rise of temperature leads to the recession and stuffing of the vortex system (as shown in Figure 3); the geopotential height in the polar regions changes from a negative anomaly to a positive one (as shown in Figure 4).

2. Along the $40^{\circ} \mathrm{N}$ latitude circle, during the periods of positive and negative phases of $\mathrm{AO}$, the temperature changes from pleions into negative meions, and correspondingly, the geopotential height field also changes from a positive anomaly belt into a negative one.

3. During the period of the positive phase of AO in 1993 January, the barometric gradient from the central and high latitudes to the Arctic region increases abnormally, which leads to the abnormal enhancement of western wind circulation, the prevalence of zonal circulation surrounding the polar regions, and the effective separation of cold air in the Arctic area. During the period of the negative phase of AO in 1977 January, the zonal circulation of the central and high latitudes surrounding the polar regions collapses and the polar cyclone is split and separated into the North American Great Lakes cold vortex and the East Asia cold vortex, which leads to frequent cold waves in North America, Asia, and most areas of the central latitudes in the northern hemisphere, resulting in a cold climate.

\subsection{Cause and Significance of Abnormal Changes in Polar Temperatures}

If the temperature changes from meions into pleions during the periods of the positive and negative phases of $\mathrm{AO}$ in the Arctic regions, then what is the significance of the opposite changes of the polar temperature symbols? What factors cause the opposite changes in polar temperature symbols? What is the relation between the changes in temperature and in the pressure field?

1. If the average temperature of the air column between the isobaric surface $\mathrm{P}_{1}$ and $\mathrm{P}_{2}$ is $T_{\mathrm{bm}}$, based on the integral equation of statics, then the geopotential thickness between the two isobaric surfaces $\Phi_{2}-\Phi_{1}$ is calculated as follows:

$$
\Phi_{2}-\Phi_{1}=-R \int_{p_{1}}^{p_{2}} T d \operatorname{In} P=R T_{\mathrm{bm}} \operatorname{In} \frac{p_{1}}{p_{2}} .
$$

Based on this, the change in geopotential thickness $\Phi_{2}-\Phi_{1}$ between the two isobaric surfaces is determined by the average temperature of air column $T_{\mathrm{bm}}$, and thus the abnormal change in polar temperature will lead to changes in the polar vortex strength. When the polar temperature rises abnormally, the $500 \mathrm{hPa}$ geopotential height will increase, the polar vortex will weaken or even become high atmospheric pressure through filling, and at the negative phase of AO in 1977 January, the polar temperature was abnormally high, thus the polar vortex disappeared and a high-pressure center took over. When the polar temperature is abnormally low, the $500 \mathrm{hPa}$ geopotential height will decrease and the polar vortex will deepen; for example, at the positive phase of AO in 1993 January, the polar vortex deepened with the decline of polar temperature, the barometric gradient from the central and high latitudes to the Arctic region was abnormally large; and the west wind circulation strengthened and zonal circulation surrounding the pole prevailed.

2. With the change in the pressure field, the distribution of temperature advection dominated by the high-pressure ridge changes accordingly and thus affects the temperature distribution pattern. For example, at the positive phase of AO in 1993 January, it was only a shallow ridge on the Atlantic coast of southern Europe and the Pacific coast of western North America and the warming caused by the warm advection behind the ridge was mainly found in southern Europe and southern North America; at the negative phase of AO in 1977 January, the shallow ridge on the Atlantic coast of southern Europe and the Pacific coast of western North America strengthened and advanced northward (see Figure 3), and the warming caused by the warm advection behind the ridge affected not only southern Europe and western North America but also the Arctic regions (see Figure 3), which was a principal element for the warming of the Arctic regions. The cold advection in front of the ridge guided the cold air in the Arctic region to advance southward, which deepened the East Asia major trough into the East Asia cold vortex, as well as the North American Great Lakes trough of low pressure into the Great Lakes cold vortex, and it was these two deep, cold vortices that led to the abnormal cold in the central latitudes of the northern hemisphere.

The above two points indicate that although the pressure field is determined by air temperature, when the situation of the pressure field changes, it will soon have an adverse effect on the distribution of the temperature field. Therefore, the temperature field inside the atmospheric system tends to be in an equilibrium state with rapid mutual adaptation. The equilibrium may be broken only after inputting external additional heat, after which a climate anomaly will be caused.

3. The heat sources of the atmosphere system mainly originate from solar radiation, the solar radiation reflected by the Earth, and the long-wave radiation of the Earth. In addition, the atmosphere is heated by the transferred sensible and latent heats from the surface into the atmosphere, the latter two of which are caused mainly by solar radiation. Therefore the main heat source of the Earth's atmosphere system is solar radiation.

With the continuously deepening development of the study of solar radiation, many scholars have gradually recognized that the solar radiation affecting the climate of the Earth includes two aspects: visible light and short-wavelength radiation and eruption of solar energetic particle flow.

Solar visible light and short-wavelength radiation are the determining factors in the formation of the Earth's climate, but their anomalous changes are small, accounting only for about $1 \%$ of the total solar radiation, which is not sufficient to stimulate the Earth's climate to oscillate sharply. Previous studies have shown that the high-energy particle flow during the solar explosion period is several million times as large as that in the quiet period, and it is the most important factor causing the anomalous oscillation of the Earth's climate. 
Table 4

Power Spectrum and Test Spectrum of the AO Index 1700-2010

\begin{tabular}{lccccccccc}
\hline \hline Wave Number & 0 & 1 & 2 & 3 & $\mathbf{4}$ & 5 & 6 & 7 & 8 \\
\hline Wavelength & $\infty$ & $\mathbf{1 1 0}$ & 44 & 29 & $\mathbf{2 2}$ & 17 & 14 & 12 & 11 \\
Spectral density & 0.009 & $\mathbf{0 . 4 5 9}$ & 0.082 & 0.038 & $\mathbf{0 . 2 3 3}$ & 0.053 & 0.027 & 0.020 & 0.002 \\
Test spectrum & 0.686 & $\mathbf{0 . 3 7 9}$ & 0.162 & 0.083 & $\mathbf{0 . 0 4 9}$ & 0.033 & 0.023 & 0.017 & 0.013 \\
Amplitude & 2.000 & $\mathbf{0 . 5 9 2}$ & 0.268 & 0.216 & $\mathbf{0 . 3 5 7}$ & 0.153 & 0.162 & 0.054 & 0.042 \\
Initial phase angle & 0.000 & $\mathbf{0 . 5 1 0}$ & 0.867 & -0.265 & $\mathbf{- 1 . 5 3}$ & -1.27 & 0.338 & 0.051 & 1.210 \\
\hline
\end{tabular}

The solar explosion period is also the anomaly enhancement period of the solar magnetic field. In addition, the solar highenergy particle flow moves outward around the solar magnetic field lines, thus the changes in solar magnetic field intensity and magnetic field direction are the key factors of the solar activity influencing the climate of the Earth. In addition, the changes of the solar magnetic field direction may exert an influence on the Earth's magnetosphere forms, i.e., when the solar magnetic field moves toward the south, the interplanetary magnetic field lines link to the Earth's magnetic field lines, the Earth's magnetosphere becomes an open magnetosphere, and large amounts of plasma carried by solar wind flow into the Earth's magnetosphere The charged particles which are trapped in the Earth's magnetic field travel between the north and south poles, around the magnetic lines of force under Lorentz force, thus releasing energy and heating the atmosphere. By contrast, when the solar magnetic field moves toward the north, the Earth's magnetosphere closes and large amounts of plasma carried by the solar wind glide across the periphery of the Earth's magnetosphere and it becomes difficult for them to enter the Earth's magnetosphere. Therefore, the periods in which the solar magnetic field moves toward the south and the north correspond to those in which the Earth's magnetosphere gains abnormally more or abnormally less solar radiation energy. Clearly, this condition is crucial for the climate of the Earth. High-energy particle flow not only influences the upper atmosphere in the polar regions, but also contacts the lower atmosphere directly and heats it in the polar regions. Therefore, the polar regions are particularly sensitive to solar explosions. This paper investigates the influences of solar activity on AO from the two aspects of the changes in solar magnetic field intensity and the direction of the magnetic field.

\section{PERIODICITY OF ANOMALOUS CHANGES OF AO AND ITS RELATION TO SOLAR ACTIVITY}

There is a significant $80-110$ yr century cycle and a quasi $22 \mathrm{yr}$ cycle of the changes in solar magnetic field and the direction of the magnetic field. Due to the fact that the changes in atmospheric temperature in the arctic regions are particularly sensitive to solar explosions, logically, AO should have periodic changes with a scale similar to the solar activity. Is this the actual situation? Indeed it is. This paper mainly expounds the periodicity of AO and its close relationship with solar activity.

\subsection{Century Cycle of $A O$ and its Relationship to the Century Cycle of the Solar Field}

The power spectrum analysis of the time series of the AO indices from 1889 to 2013 has been completed and a significance test (reliability $\alpha=0.05$ ) has been conducted. The computational scheme is shown in Qu et al. (2003). As may be seen from Table 4 (boldface), the power spectral density of the AO index in the second line is located at the first wave (the corresponding cycle of which is $110 \mathrm{yr}$ ) and the fourth wave (corresponding cycle of $22 \mathrm{yr}$ ). These values are significantly greater than the red-noise testing spectral values in the third line, thus they pass the significance test.

This signifies that the 110 cycle corresponding to the first wave and $22 \mathrm{yr}$ cycle corresponding to the $\mathrm{f}$ wave are both significant cycles of $\mathrm{AO}$.

According to the fifth and the sixth lines in the table, it may be seen that when the $110 \mathrm{yr}$ periodic wave amplitude $A$ is 0.592 , the initial phase angle is $0.51 \mathrm{rad}$. The equation for the $110 \mathrm{yr}$ periodic component $C_{110}(i)$ may be written as follows:

$$
C_{110}(i)=0.592 \sin \left(\frac{2 \pi}{110}+0.51\right), \quad(i=1,2, \ldots \ldots 311),
$$

where $l$ may be regarded as the wave number, namely the order of the waves, and $\mathrm{m}$ represents the maximum a posteriori, namely the total number of partial waves.

After drawing the five-year average moving column line of the abnormal values of the AO indices in January and the $110 \mathrm{yr}$ periodic component curve in Table 4, and comparing the AO index curve (column line) with the $110 \mathrm{yr}$ cycle component curve (solid line), it may be seen that the variation tendencies of the two are basically the same. In addition, their wave crests and wave troughs are also basically the same, indicating that the $110 \mathrm{yr}$ cycle has significant cyclical characteristics contained in the century changes of the AO indices, and the $110 \mathrm{yr}$ cycle may be seen as the variation tendency of $\mathrm{AO}$ during the period of 1900 to 2013.

(1) The right side of the equation for the first type of solar activity cycle, with 110 separate $C_{\mathrm{R} 110}$, is

$$
C_{R 110}(i)=15.17 \sin \left(\frac{2 \pi}{110} i-1.39\right) \quad(i=1,2, \ldots \ldots 311) .
$$

In order to facilitate the analysis of the relationship between the solar activity century cycle and AO century cycle, we may superimpose the quasi $110 \mathrm{yr}$ cycle curve of the solar field (dotted line) in Figure 6 . The $110 \mathrm{yr}$ cycle of the solar field is calculated according to type (5) in Figure 6. Then from Figure 6 we may see that the crests and troughs of the quasi $110 \mathrm{yr}$ century cycle of the solar activity are contrary to those of the quasi $110 \mathrm{yr}$ century cycle of $\mathrm{AO}$, and the quasi $110 \mathrm{yr}$ century cycle of the solar activity changes ahead of time. Therefore it may be incurred that the solar activity inspires the century cyclical change in $\mathrm{AO}$.

In order to facilitate the analysis of the effects of the solar activity on the interdecadal cyclical changes of AO, we may first filter the century cycle from the sequence of the $\mathrm{AO}$ indices. After subtracting the $110 \mathrm{yr}$ cycle from the sequence of original 


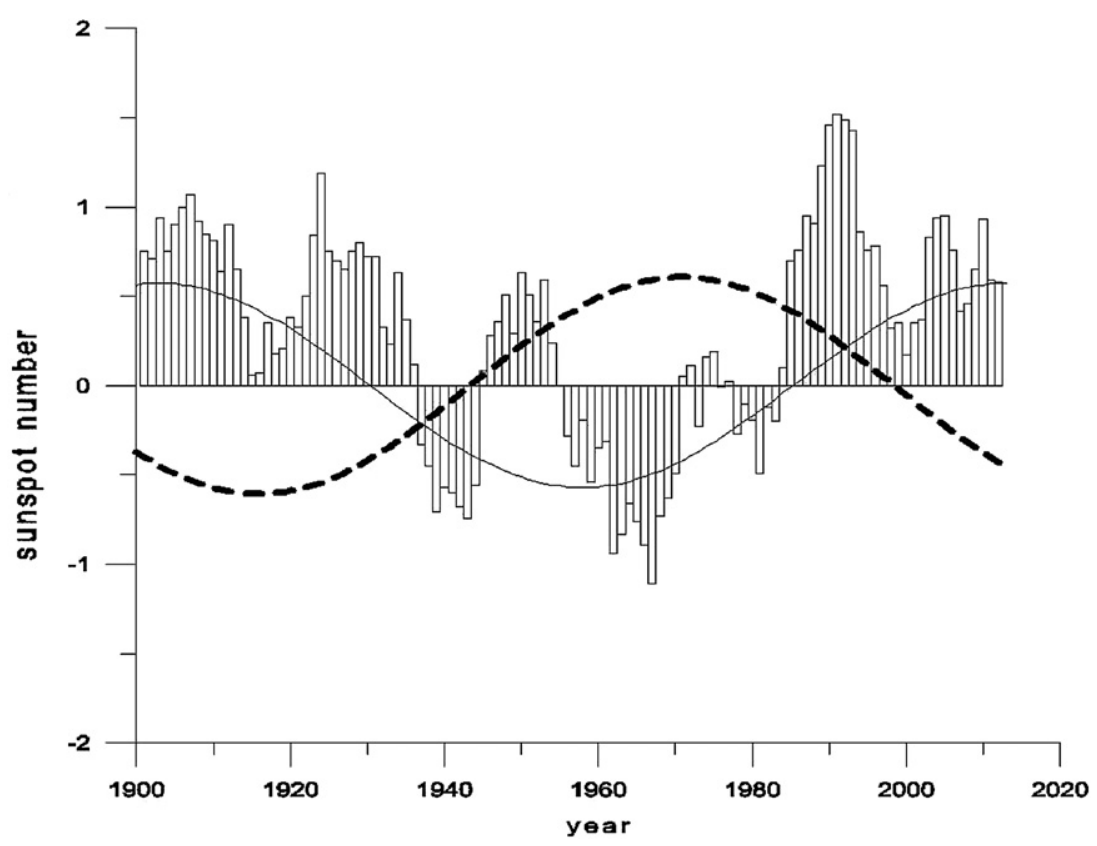

Figure 6. Five-year average moving column line of AO indices in January and quasi $110 \mathrm{yr} 22$ year periodic component curve of AO in January (thin line) and quasi $22 \mathrm{yr}$ cycle curve of the solar magnetic field MI (thick dashed line).

$\mathrm{AO}$ indexes $\mathrm{AO}(i)$, we may obtain the sequence of $\mathrm{AO}$ indices without century cycle $y(i)$ :

$$
y(i)=A O(i)-C_{110}(i) .
$$

Then we may obtain the variance of the sequence of original $\mathrm{AO}$ indices:

$$
s^{2}=\frac{1}{n} \sum_{i=1}^{n}(A O(i)-\overline{A O})^{2}=0.3481
$$

where $\overline{A O}$ represents the medium value of the $\mathrm{AO}$ indices in January during the period of 1889 to 2013 . The variance of the AO index sequence in January after filtering the changes in the century cycle is $S_{1}{ }^{2}=0.441$, then the variance contribution $\mathrm{SS}$ of changes in century cycle to $\mathrm{AO}$ indexes is determined as follows:

$$
\begin{aligned}
\mathrm{SS} & =\left(\left(S^{2}-S_{1}^{2}\right) / S^{2}\right) \times 100 \% \\
& =((0.3481-0.441) / 0.3481) \times 100 \%=44.4 \% .
\end{aligned}
$$

Therefore, it may be observed that the changes in the 110 year cycle are a very important feature of the changes in the $\mathrm{AO}$ index century, and the extent of their impact surpasses $44 \%$ of the abnormal changes of the total AO indexes.

Generally speaking, due to the fact that the range of the data used in this study is not sufficiently broad, it is not highly credible for analyzing the changes in the 88 year cycle of $\mathrm{AO}(i)$ according to the data from the period of 1889-2013. However, from Table 4, it may be seen that the factual data of AO (i) are basically the same as the $110 \mathrm{yr}$ cycle curve, indicating that the 88 yr cycle basically reflects the variation tendency of the $\mathrm{AO}(i)$ for a period of more than $100 \mathrm{yr}$, thus we may regard the quasi $110 \mathrm{yr}$ cycle as the trend term of the sequence of $\mathrm{AO}(i)$ during the period of 1889-2013.

\subsection{2 yr Cycle of $A O$ and its Relation to the Solar Magnetic Field MI}

We conducted the analysis of the power spectrum of the sequence of $\mathrm{AO}$ indices without the century cycle for the period of 1889-2013 and performed a significance test when the reliability $\alpha$ is 0.05 , the numerical procedure for which may be seen in Qu et al. (2003). From Figure 7, it may be seen that the column line in the estimated power spectrum of the AO indices is highest in the fourth wave and that it passes the significance test when the reliability is 0.05 . This signifies that the quasi $22 \mathrm{yr}$ cycle corresponding to the fourth wave is the significant cycle of AO. Although the second wave also passes the significance test when the reliability is 0.05 , the crest is indistinctive, indicating that the $88 \mathrm{yr}$ cycle has been basically filtered out from the $\mathrm{AO}$ (i). According to Qu et al. (2003), we may see that when the $22 \mathrm{yr}$ periodic wave amplitude $A$ is 0.357 and the initial phase angle is $1.53 \mathrm{rad}$, then the equation for the periodic component of the $22 \mathrm{yr} C_{22}(i)$ may be written as follows:

$$
\begin{aligned}
C_{22}(i)= & \sum_{i=1}^{m} A_{l} \sin \left(\frac{2 \pi}{T_{l}} i+\varphi_{l}\right)=0.357 \\
& \times \sin \left(\frac{2 \pi}{22} i-1.53\right)(i=1,2, \ldots \ldots 101),
\end{aligned}
$$

where $l$ may be regarded as the wave number, namely the ranking of the wave, and $m$ represents the maximum a posteriori, namely the total number of partial waves.

After drawing the abnormal value curve of the AO indices after filtering the variation tendency in January and the $22 \mathrm{yr}$ periodic component curve in Figure 7, and comparing the AO index curve (column line) with the $22 \mathrm{yr}$ periodic component curve (thin line), we can see that the variation tendency of the two are basically the same. In addition, their wave crests and wave troughs are also basically the same, indicating that the 22 yr cycle has significant cyclical characteristics contained in the interdecadal changes of the $\mathrm{AO}$ indices and its variance 


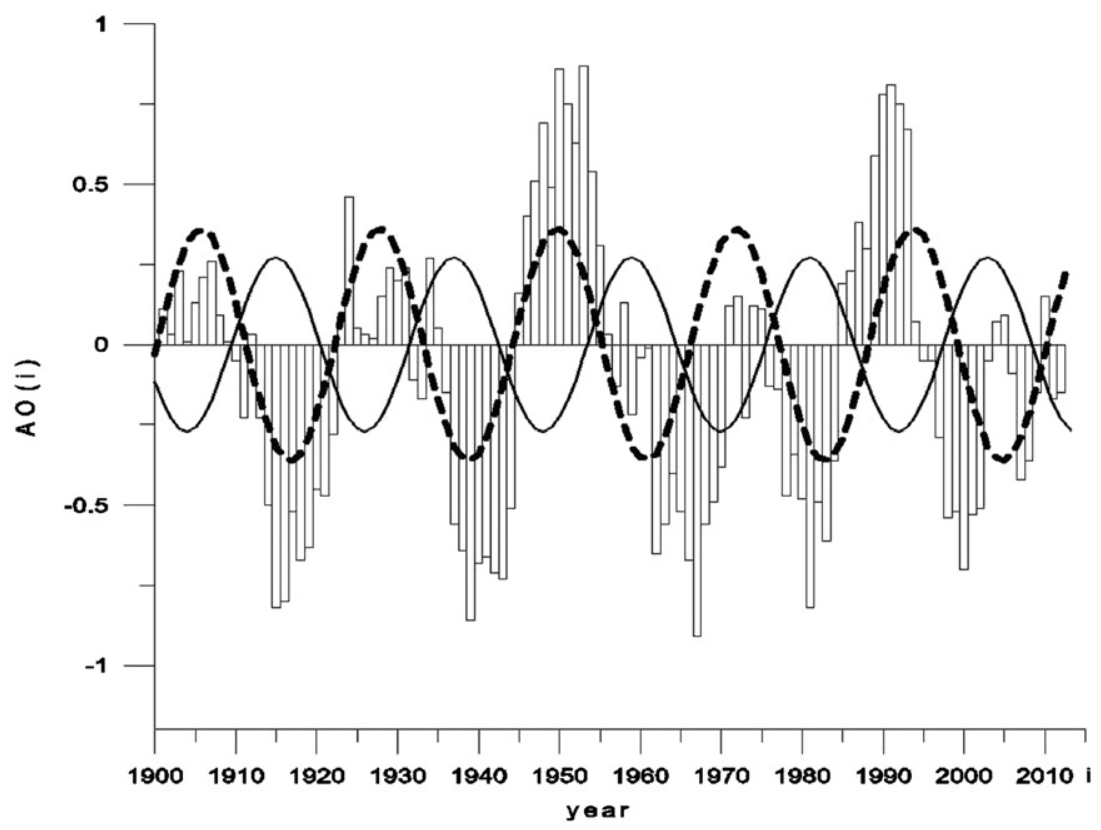

Figure 7. Column line of AO without century cycle and $22 \mathrm{yr}$ periodic component curve in January (thick dashed lines) and quasi $22 \mathrm{yr}$ cycle curve of the MI of the solar magnetic field (thin lines) in January.



Figure 8. $22 \mathrm{yr}$ periodic component curve of AO in January (bold line), MI curve of the solar magnetic field (except 150) (thin lines), and the quasi $22 \mathrm{yr}$ cycle curve of the solar magnetic field MI (thick dashed line)

contribution rate reaches $18.5 \%$, second only to the important characteristics of the interdecadal changes of the AO indices after the quasi $110 \mathrm{yr}$ cycle. After superimposing the quasi $22 \mathrm{yr}$ cycle curve of the solar magnetic field MI (thick dashed line) in Figure 8, we can see that the quasi $22 \mathrm{yr}$ cycle curve of the MI is contrary to the $22 \mathrm{yr}$ periodic component curve of the $\mathrm{AO}$ (thin line) in the variation tendency. Furthermore, the wave crests of the quasi $22 \mathrm{yr}$ cycle of the MI and wave troughs of the $22 \mathrm{yr}$ cycle of the $\mathrm{AO}$ correspond to one other and are somewhat ahead of times. This indicates that the quasi $22 \mathrm{yr}$ cycle of the MI causes the $22 \mathrm{yr}$ cycle of the AO.

In order to facilitate the analysis of the relationship between the solar activity magnetic century cycle and the AO century cycle, we may superimpose the quasi 22 yr cycle curve of the MI of the solar magnetic field (thick dashed line) in Figure 8. The $22 \mathrm{yr}$ cycle of the MI of the solar magnetic field is calculated according to type (7), the drawing in Figure 8. Then from Figure 6 we may see that the crests and troughs of the quasi $22 \mathrm{yr}$ century cycle of the solar magnetic activity are contrary to those of the quasi $22 \mathrm{yr}$ century cycle of $\mathrm{AO}$, and the quasi $22 \mathrm{yr}$ century cycle of the solar activity magnetic changes ahead of time. Therefore it may be inferred that the solar activity inspires the century cyclical change of AO.

In order to facilitate the analysis of the relationship between solar activity and the periodic changes in $\mathrm{AO}$, we superimpose the $22 \mathrm{yr}$ periodic component currve of the $\mathrm{AO}$ (thick dashed 
line) and the MI curve of the solar magnetic field (thin line) in Figure 8, then compare the two. We can see that their respective variation tendencies are basically contrary to one another, as are their wave crests and wave troughs. In addition, the wave crests and wave troughs of the solar magnetic field MI curve are ahead of the $22 \mathrm{yr}$ periodic component curve of $\mathrm{AO}$ and the tropism of the wave crests and wave troughs of the two are relatively fixed, thus showing that there is a close inverse relationship between solar activities (especially the changes in the MI of the solar magnetic field) and the changes in the $22 \mathrm{yr}$ cycle in January. After superimposing the quasi 22 yr cycle curve of the MI of the solar magnetic field (red dotted line) in Figure 8, we may see that the quasi 22 y cycle curve of the MI is basically the same as the wave crests and wave troughs of the solar magnetic field MI curve, thus indicating that the quasi $22 \mathrm{yr}$ cycle of the solar magnetic field MI is an inherent oscillation cycle of the solar magnetic field.

A previous study performed by one of the authors of the present one pointed out that when the solar magnetic field faces south, the solar magnetic field MI curve is located in the section from wave trough to wave crest. At this time, under perturbation from the various wavelengths of the solar magnetic field, it may easily maintain its southward component, thus the opportunities to reconnect with the magnetic field line of the Earth increase, in turn causing a large number of charged particles to enter the magnetosphere of the Earth and heat its atmosphere. From Figure 8 , we may see that the $22 \mathrm{yr}$ periodic component curve of AO in January (thick dashed line) is from wave crest to wave trough when the solar magnetic field faces south, i.e., the solar mass ejection heats the Earth's atmosphere in the Arctic region, then the atmosphere of the region becomes warm, corresponding to the negative phase period of AO.

By contrast, when the solar magnetic field faces north, the MI curve of the solar magnetic field is from wave crest to wave trough. At this time, under perturbation from the various wavelengths of the solar magnetic field, it cannot easily maintain its southward component, thus the opportunities to reconnect with the magnetic field line of the Earth decrease, in turn causing a large number of charged particles to slide over the periphery of the magnetosphere of the Earth, and its atmosphere cannot be heated. From Figure 8, we may see that the 22 year periodic component curve of AO in January (thin line) is from wave trough to wave crest when the solar magnetic field faces north, i.e., the solar mass ejection slides over the Earth, thus the atmosphere in the polar region cannot be heated and the atmosphere of the region remains cold, corresponding to the positive phase period of AO.

\section{CONCLUSIONS}

In this study, based on a comparative analysis of geopotential height, the geopotential height anomaly, and the monthly mean temperature and anomaly of the northern hemisphere $500 \mathrm{hPa}$ during the positive and negative phases of $\mathrm{AO}$, one can see that the abnormal warming period of the Arctic region corresponds to the negative phase of $\mathrm{AO}$, while the anomalous cold period of the Arctic region corresponds to the positive phase of $\mathrm{AO}$, thus showing that the abnormal changes in the Arctic region determine the signs of anomalies of AO.

After performing analysis using the successive filtering method, one can see that the AO phenomenon occurring in January shows clear a quasi $110 \mathrm{yr}$ century cycle and quasi $22 \mathrm{yr}$ decadal cycle which are closely related to solar activities. The specific observations are as follows.

1. The AO phenomenon occurring in January has a clear quasi $110 \mathrm{yr}$ century cycle that is closely related to solar activities. The variance contribution rate of the quasi $110 \mathrm{yr}$ century cycle for the $\mathrm{AO}$ indices reached up to $44.4 \%$, which is one of the most significant century change characteristics in the $\mathrm{AO}$ phenomenon occurring in January.

2. The spectral analysis results show that the AO indices in January, after filtering the changes in the $88 \mathrm{yr}$ century cycle, have a significant $22 \mathrm{yr}$ cycle and their variance contribution rate reached up to $18.5 \%$, preceded only by the important characteristics of the interdecadal changes in the AO indices after the quasi $110 \mathrm{yr}$ century cycle. The comparative analysis shows that a close inverse relationship exists between solar activities (especially solar magnetic field index changes) and changes in the $22 \mathrm{yr}$ cycle of the AO in January, and that the two trends are substantially opposite from each other. In other words, in most cases when the solar magnetic index (MI) rises from the lowest value, then the AO will drop from the highest value and when the MI drops from the highest value, $\mathrm{AO}$ will rise from the lowest value.

In summary, the variance contribution rate of the changes in the quasi $110 \mathrm{yr}$ century cycle and quasi $22 \mathrm{yr}$ decadal cycle for the $\mathrm{AO}$ reached up to $62.9 \%$, which indicates that solar activity is an important factor driving the AO.

This work is funded by the Global Change Research Program of China (2010CB951403) and the National Science Foundation of China: Arctic Sea ice and the coupling of the upper ocean circulation changes and climate effect (41330960) and (41376008).

\section{REFERENCES}

Christensen, E. F. 1993, Energy, 18, 1273

Christensen, E. F., \& Lassen, K. 1991, Sci, 254, 698

Dong, S. L. 1997, AcApS, 17, 86

Gong, D. Y., Zhu, J. H., \& Wang, S. W. 2002, Chin. Bull. Sci., 47, 546

Lean, J., Beer, J., \& Bradley, R. 1995, GeoRL, 22, 3195

Peristykh, A. N., \& Damon, P. E. 2003, JGR, 108, 1003

Qu, W. Z., Deng, S. G., Huang, F., \& Zhao, X. 2004, ChJG, 47, 398

Qu, W. Z., Huang, F., \& Zhao, J. P. 2005, Acta Oceanol. Sinica, 27, 215

Qu, W. Z., Huang, F., Zhao, J. P., et al. 2007, ChJG, 50, 1304

Qu, W. Z., Wang, L., Huang, F., \& Liu, Y. C. 2003, Jouc, 33, 329

Qu, W. Z., Zhao, J., Huang, F., \& Deng, S. 2012, AJ, 144, 6

Rigor, I. G., Colony, R. L., \& Martin, S. 2000, J. Climate, 13, 896

Shi, Z. X., \& Wang, J. X. 1994, ChJSS, 14, 22

Thompson, D. W. J., \& Hurrell, J. W. 2002, The AGU Monograph on The NAO, 126,157

Thompson, D. W. J., \& Wallace, J. M. 1998, GeoRL, 25, 1297

Thompson, D. W. J., Wallace, J. M., \& Hegerl, G. C. 2000, JCl, 13, 1018

Xia, Y., \& Jin, W. 2001, PABei, 19, 34

Xuong, G. M., Chen, Q. L., Jiang, K., et al. 2012, J. Chengdu Univ. Inform. Technol., 27, 273

Zhang, D. H., \& Xiao, Z. 2000, Acta Sci. Nat. Univ. Pekinesis, 36, 414

Zhang, Z. D., Lin, J., Chen, J., \& Wu, N. 1998, PABei, 16, 196 